\title{
SIMBOLISMO SOCIAL ASOCIADO Y ACTITUD MANIPULATIVA EN NIÑOS AMAZÓNICOS PERUANOS
}

\author{
SIMBOLISMO SOCIAL ASSOCIADO E ATITUDE MANIPULATIVA EM \\ CRIANÇAS AMAZÔNICAS PERUANAS \\ ASSOCIATED SOCIAL STMBOLISM AND MANIPULATIVE
ATTITUDES TOWARDS CHILDREN FROM THE PERUVIAN AMAZON
}

\section{Luis Fidel Abregú Tueros ${ }^{1}$, Jussara María Rosa Mendes² e Lourdes Abregú Arroyo ${ }^{3}$}

${ }^{1}$ Universidad Nacional Agraria de la Selva, Tingo María, Perú

${ }^{2}$ Universidade Federal do Rio Grande do Sul, Porto Alegre/RS, Brasil

${ }^{3}$ Hospital Nacional EsSalud “E. Rebagliati” Lima, Perú

RESUMEN: El Simbolismo Social Asociado (SSA) y la Actitud Manipulativa (AM) están presentes en la mayoría de niños de nueve años de edad de la Amazonía peruana con desarrollo intelectual adecuado (71.2\%). El estudio se realizó en 52 niños y sus padres mediante entrevistas en pequeños grupos, aplicándose la prueba TMP y dos guías de entrevista (niños-padres). Los resultados señalan correspondencia significativa entre la preferencia (niños) y la decisión de compra del producto simbolizado (padres), y el 78,5\% elegían la misma marca que sus pares. Las dificultades de comprensión del contenido material de la publicidad (CMP) involucran al 78,9\% (mayor en área urbana). Existe interdependencia significativa entre AM (niños) y reacciones de compra (padres) y alta discordancia entre respuestas (niños-padres). Educar en la comprensión del CMP a partir de nueve años, podría disminuir las consecuencias del SSA mediante programas psicosociales para la salud mental escolar.

PALABRAS CLAVE: Salud Mental; Salud Escolar; Factor Psicosocial; Simbolismo Social, Actitud Manipulativa.

RESUMO: O Simbolismo Social Associado (SSA) e a Atitude Manipuladora (AM) estão presentes na maioria das crianças de nove anos de idade, da Amazônia Peruana, com desenvolvimento intelectual adequado (71,2\%). O estudo foi realizado com 52 crianças e seus pais, por meio de entrevistas em pequenos grupos, aplicando-se o teste TMP e dois roteiros de entrevista (crianças-pais). Os resultados indicam correspondência significativa entre a preferência (crianças) e a decisão de compra do produto simbolizado (pais), e 78,5\% escolheram a mesma marca que seus amigos. As dificuldades de compreensão do conteúdo material da publicidade (CMP) envolvem 78,9\% (maior na área urbana). Existe interdependência significativa entre AM (crianças) e reações de compra (pais) e alta discordância entre respostas (crianças-pais). Educar, na compreensão do CMP, a partir de nove anos, poderia diminuir as consequências do SSA através de programas psicossociais para a saúde mental escolar.

PALAVRAS-CHAVE: Saúde Mental; Saúde Escolar; Fator Psicossocial; Simbolismo Social, Atitude Manipulativa.

ABSTRACT: Associated social symbolism (ASS) and the manipulative attitude (MA) are present in most nine-yearold children from the Peruvian Amazon with adequate intellectual development (71.2\%). The study was conducted in 52 children and their parents through interviews in small groups, applying the TMP test and two interview guides (child-parents). The results indicated significant correspondence between the preference (children) and the decision to purchase the product symbolized (parents), and $78.5 \%$ chose the same brand as their peers. Difficulties in understanding the content of advertising material (CAM) involve $78.9 \%$ (higher in urban area). There is significant interdependence between MA (children) and buying reactions (parents) and high discordance between responses (children-parents). Educate in the understanding of the CAM from nine years, could reduce the consequences of ASS through psychosocial programs for school mental health.

KEYWORDS: Mental Health; Educational Health; Psychological Factor; Social Symbolism; Manipulative Attitudes. 


\section{Introducción}

Los aspectos psicosociales del desarrollo infantil son fundamentales para determinar la orientación educativa y el apoyo social en la salud mental, particularmente para el desarrollo emocional y la prevención en las diferentes etapas evolutivas, que son metas prioritarias para los organismos internacionales como la UNICEF, UNESCO y Asociación Mundial para la Salud Mental Infantil -WACMH- (Marcos \& Carrillo, 2014), quiénes recomiendan que las intervenciones deben ser multidisciplinarias cuando se trate de actitudes simbólicas y manipulativas, por ser dificultades tanto del comportamiento, de las emociones como de las relaciones interpersonales en la niñez (Addis \& Holbrook, 2006; Marcos \& Carrillo, 2014; Martínez, 2014; Petty, 2006; Ruiz, 2014).

Siendo la Actitud Simbólica (AS) la predisposición organizada a través de la experiencia que incita al individuo reaccionar frente a las personas, las cosas, las actividades y las ideas; respondiendo en las dimensiones cognitiva (conocimiento, creencia, valor y pensamiento), conductual y afectiva (Addis \& Holbrook, 2006; Marcos \& Carrillo, 2014; Petty, 2006) y cuyo elemento común es el simbolismo social asociado (SSA). Mientras que la Actitud Manipulativa (AM) o coacción psicológica oculta, es la influencia psicológica consciente no psicopática que aparece en todo los niveles de la interacción social e interpersonal, como puede ser el seno familiar; apareciendo cuando los hijos/as influyen en la reacción de sus padres (en adelante genérico de padres y madres) para obtener un objeto o recompensa deseada (Bereczkei, 2015; Decety, Jackson, Sammerville, Chaminade, \& Meltzoff, 2004); es inherente a diferentes culturas y condiciones históricas, que tiene concordancia con la presencia de habilidades de supervisión vinculantes con la toma de decisiones y el seguimiento de conductas orientadas a la búsqueda de recompensas, cuyas funciones neurológicas corresponden a la corteza órbito frontal anterior izquierda del cerebro (Berne, 2011; Dotsenko, 2003; Monich \& Matveeva, 2012; Zhumagaliyeva \& Barabanova, 2014).

\section{Enfoque psicosocial del simbolismo desde la perspectiva cognitivo-afectiva}

El enfoque psicosocial del simbolismo no es reciente pero es poco explorado, proponiéndose situar el proceso de simbolización en la etapa infantil en los planos social y personal desde la perspectiva cognitivo-afectiva. Aquí el simbolismo y su representación social no sólo se relacionan con las ideas, sino también con los comportamientos; conectándose hacia las prácticas colectivas y el abordaje simbólico de la reciprocidad y la cohesión grupal (Malinowski, 2001).

Después de la etapa infantil se inicia el periodo en que el conocimiento se apoya en las emociones, y así la simbolización se presenta como una actividad constante a lo largo de la vida. Con el acceso del sujeto al universo simbólico establecido en su realidad cultural, ocurre la vinculación entre el deseo y la realidad social, donde incluso los conflictos personales, las contradicciones emocionales, la ansiedad y las frustraciones, como los modelos de identificación; constituyen las fuentes motivacionales y los mecanismos para la formación simbólica (Anzieu, 1981; Furth, 1992). Reconociéndose al simbolismo por su polisemia e incluso por su arbitrariedad, que tiende a manifestarse en distintas formas y en función de los fenómenos, del momento histórico-cultural como de los modelos de interpretación de los mismos, quedando configurado el simbolismo por sus determinantes sociales (Barbeta, 2015, p.166). 
En este proceso de simbolización los individuos tienden quedarse en sus deseos e intereses particulares subordinados al nivel holista de lo colectivo, entendiéndose la producción simbólica como algo inherente a la vida social.

Entre tanto, los símbolos pueden ser objetos, acontecimientos y emociones, que tienen como papel principal crear significados, conducir información a los sujetos sobre los procesos externos que los ponen en juego y, en definitiva organizar las experiencias, los procesos sociales y psicológicos (Barbeta, 2015, p.175, Schutz, 1974). En el caso de las mercancías, por ejemplo, los símbolos son elementos para el intercambio comunicativo que no sólo implican la diferenciación entre sujetos y grupos sociales, sino entre la organización significativa del medio social y la integración/exclusión del mismo, donde el consumo de bienes simbólicos refleja la intensidad de las relaciones sociales (Douglas \& Baron, 1990).

Quedando establecido el simbolismo, como una matriz de acciones intersubjetivas y conocimientos compartidos que se configuran en la práctica social, posibilitando la realización de experiencias significativas y la comunicación entre sujetos de distintos niveles de la realidad: vida cotidiana, experiencias religiosas, fantasías, etc. (Schutz, 1974, p. 296).

Por ende, las principales características del simbolismo desde la perspectiva cognitivo-afectiva son: (a) creaciones y expresiones de las relaciones y procesos sociales que se abren a la realidad extralingüística; (b) asociaciones entre procesos sociales que para aprehenderlos son necesarias la interpretación; (c) signos polisémicos; (d) sistemas de símbolos socioculturales e históricos específicos (Barbeta, 2015, p. 178). Considerando a las formaciones simbólicas como las respuestas personales afectivas hacia los contextos socioculturales e interpersonales (Ortí, 1994) y su estrecha relación entre las estructuras simbólicas (cognitivas, de percepción y representación) y las estructuras sociales, por ello todo los hechos sociales son entendidos como las formas de comunicación simbólica con capacidad de dominio de unos grupos sociales sobre otros; mientras que el poder simbólico está expresando, reproduciendo y ocultando los procesos sociales reales (Barbeta, 2015; Lévi-Strauss, 1987)

De ahí que los sistemas simbólicos tienen como papel principal estructurar concepciones de los medios sociales concretos, representarlos y clasificarlos; por ejemplo, los tabúes alimenticios expresan simbólicamente las clasificaciones prácticas y la distinción entre las categorías sociales (Douglas \& Baron, 1990); y las imágenes del cuerpo humano, en función de los individuos y medios sociales en los que se encuentran insertos, se utilizan de una u otra forma reflejando de modo simbólico el espacio social y la experiencia que tienen los sujetos. Manteniéndose un vínculo entre los medios sociales empíricos en los cuales los individuos viven y se desarrollan, y las formas del pensamiento y la conducta social, que no serían sino la expresión simbólica de dicho vínculo.

\section{Porqué del estudio en niños y niñas de nueve años de edad}

Según Piaget (1970), las dificultades para reconocer cognitivamente la intención persuasiva de la publicidad están presentes en niños hasta los ocho años de edad (en adelante genérico de niños y niñas), quiénes se ubican en la etapa operacional concreta del desarrollo cognitivo, un grupo etario vulnerable porque sus pensamientos reflexivos son limitados (Hastings et al., 2003; Kunkel, 2010; Lawlor \& Prothero, 2003; Rozendaal, Buijzen, \& Valkenburg, 2011; Wartella, 2009; Young, 2015); es decir, cuanto más joven sea el niño, 
su actividad representativa estaría más subordinada a las imágenes y menos hacia los mensajes verbales (Aktaş, 2006; Lawlor \& Prothero, 2010; Nunes, 1998; Rozendaal et al., 2011; Šramová, 2014). En cambio, en niños de nueve años de edad en quiénes se verifican, (a) teóricamente la capacidad de comprensión de la intensión persuasiva estaría mejor desarrollada y consecuentemente tanto el SSA como la AM ya estarían superadas; (b) la ausencia del SSA y la AM serían similares según el género, el número de hermanos y el área geográfica. Además, como señalan Carona, Weiss, Harris y Catron (2006) la calidad del reporte de los padres sobre el seguimiento de conductas de búsqueda de recompensas de los hijos/as de nueve años de edad en adelante tienen validez predictiva sobre la internalización y externalización de AM.

Sobre los antecedentes en simbolismo social, Fenollar y Ruiz (2006) y Tsai (2005) señalan que son características propias de una cultura de consumo debido a la intermediación externa (publicidad televisiva y virtual) e interna: hedonismo (la expresión del deseo de posesión de un producto como una forma de mostrar ante el resto un estatus o éxito social), resultando los mayores generadores del comportamiento de compra hedonista debido a los contenidos simbólicos televisivos, donde los alimentos de necesidad saludables son muchas veces estigmatizados, mientras que la comida poco saludable es mejor valorada (Elliot, 2014). Al respecto, la WHO (2010) señala que en la última década la comercialización de alimentos ha sido un problema que viene afectando principalmente la salud en los niños, porque tiene impacto en el desarrollo emocional por el alto nivel de materialismo en los anuncios y entre otras consecuencias, porque influye negativamente en la autoestima, en la orientación de los valores y en las actitudes (Šramová, 2014).

Los estudios en el contexto familiar señalan que el comportamiento de los hijos puede influir de manera determinante la reacción de los padres, porque en dicha interrelación surge la influencia psicológica que conduce hacia la AM de los niños para obtener recompensas (Berne, 2011; Dotsenko, 2003; Monich \& Matveeva, 2012; Zhumagaliyeva \& Barabanova 2014); y la interpretación del comportamiento de los niños puede variar según los factores situacionales, la edad y el género (Soenens \& Vansteenkiste, 2010), aunque a los 11 y 13 años de edad el comportamiento socialmente manipulador no difiere según el género; pero la religiosidad de los padres puede favorecer el comportamiento hedonista de los hijos (Raba'ah, Turinam, Hamzah, \& Tamam, 2014). Sin embargo, tanto el egoísmo de los niños, las actitudes de tolerancia de los padres hacia sus hijos, como el dar demasiada importancia a la opinión de los pares (niños) generan un apego especial cada vez habitual (Neme \& Rodríguez, 2013; Raba'ah et al., 2014).

Por otro lado, debe considerarse que los niños son imitadores por excelencia y sensibles a la influencia de los anuncios, porque la visión que tienen sobre el mundo está en función de lo que ven y oyen, debido a que aún no distinguen entre la ficción y la realidad (Neme \& Rodríguez (2013) y Šramová (2014a). De allí que el consumo de marca está cargado de simbolismos que crean los modos de relacionarse y de qué forma ser aceptados socialmente (Holbrook, 1987; Huber, 2002; Neme \& Rodríguez, 2013; O’Shaughnessy \& O’Shaughnessy, 2002); y es moda globalizada sobre todo en países del Tercer Mundo por la repetición de marcas internacionales (Neme \& Rodríguez, 2013). Resumiendo que el simbolismo está asociado socialmente, ya que el deseo es convertido en un estado, donde el querer en lugar del tener, es la mayor necesidad de búsqueda para ser considerado y respetado socialmente (Neme \& Rodríguez, 2013; Šramová, 2014a). 
Sobre la base de los antecedentes y los presupuestos descritos acerca del simbolismo social y la presencia de estrategias manipulativas, Derbaix y Bree (1997) señalan que los niños menores de nueve años de edad prefieren escenas de acción y hazañas por eso quedan influenciados directamente por aquellos productos exhibidos en la televisión (Aktas, 2006). Pero ante la falta de deslindes claros en diversas cuestiones del simbolismo social y la presencia de AM, es necesario proponer los fundamentos de la orientación en la educación para la salud en un ámbito donde la emisión televisiva aún es limitada (Amazonía peruana) y se conocen el interés activo de los profesores de educación básica para desarrollar actividades de educación para la salud mental (Soto, 2010), y porque son conscientes que la publicidad televisiva influye negativamente el deseo de los escolares (Goldstein, 1994; Islas, Pérez, \& Hernández, 2015; Uribe, Acuña, Carrasco, \& Checura, 2006), y porque cumplen dos roles, uno educativo natural y otro orientador tanto en los escolares como en los padres de éstos (Islas et al., 2015).

Resultando pertinente promover la educación para la salud a este nivel para determinar las estrategias de prevención multidisciplinar (Gállego, Aliaga, \& Granizo, 2014; Ramasco, 2014; Ramos, Fernández, \& Carranza, 2014) orientada a reducir el fenómeno del SSA y la AM en la población escolar y desarrollar competencias emocionales para mejorar la conciencia crítica según factores sociodemográficos (Carreño, 2010; Uribe, Acuña, Carrasco, \& Checura, 2006); y como mencionan Wilson, Grizzle, Tuazon, Akyempong y Cheung (2011) continuar con la agenda de la psicología y disciplinas afines orientadas a lograr el equilibrio entre las necesidades y la salud tanto en los padres de los escolares como en los profesores, como en la implementación de actividades y programas para la protección a las consecuencias negativas del simbolismo social.

El objetivo del estudio es describir la presencia del simbolismo social asociado (SSA) y la actitud manipulativa (AM) en grupo de niños de nueve años de edad de la Amazonía peruana con desarrollo cognitivo intelectual adecuado comparados entre el área geográfica, género y número de hermanos.

\section{Materiales y métodos}

El estudio es descriptivo comparativo de corte transversal, en una muestra de escolares de nueve años de edad y sus padres de los estratos urbano y rural de la Amazonía peruana. El estrato rural, se diferencia por el ingreso familiar máximo de 87 dólares/mes y el 78\% de la población tiene educación primaria (PNUD, 2013). El grupo fue organizado seleccionando sucesivamente según criterio de conveniencia hasta completar $n=25$ niños (ene-may, 2015) y n=27 (mar-may, 2016) haciendo un total de 52 niños, tamaño superior a los señalados en varios estudios (Kähkönen, Tuorila \& Lawless; 1997; Kunkel, 20 10; Lawlor \& Prothero, 2010; Nefat \& Dujmović, 2012).

Criterios de inclusión: a) cursar el cuarto grado de primaria; b) presentar inteligencia dentro de los niveles normales para la edad evaluadas según el test no verbal de matrices a color de Raven (2000) con baremos estandarizados para la región (Abregú, 2009), esto para descartar que las dificultades para el manejo del comportamiento, de las emociones, y las relaciones interpersonales de los niños no sea debido a trastornos del desarrollo intelectual (APA, 2013; CIE-11, 2015; Salvador et al., 2011); (c) contar con autorización de los padres y, (d) participar voluntariamente a todas las sesiones del estudio. La pérdida 
de sujetos por diversos motivos fue muy alta $(84 \%)$ en ambos periodos de evaluación (por abandono, nivel intelectual inferior, entrevistas incompletas, evaluación psicométrica incompleta y ausencia en los programas sociales).

\section{Instrumentos}

1. Para formar un grupo homogéneo de niños con desarrollo cognitivo intelectual adecuado a la edad, se administraron el test no verbal señalado en el criterio b).

2. Para describir y determinar los elementos del Simbolismo Social Asociado (SSA), se empleó una guía de entrevista de 19 ítems (versión niños), que incluye pautas para formular cuestiones generales relacionadas con el tiempo de ocio y las prácticas favoritas de los niños, luego más centradas en el SSA y de cómo influyen a sus padres para conseguir el producto simbolizado o AM. Para el propósito del estudio, se eligieron al "yogur" como producto simbolizado y saludable (Kähkönen et al., 1997), que pertenece a la segunda categoría de alimentos y al grupo de publicidad asociado con diversión-aventura (González, 2008). Luego se mostraron secuencialmente una por una las láminas publicitarias que identifican al producto en cuatro marcas (A-Y; B-P; C-L; D-G), las que fueron reconocidas sin ninguna dificultad por los niños. Para determinar la presencia del SSA, se realizó la entrevista en pequeños grupos (máximo 3 niños) desarrollándose secuencialmente en el domicilio familiar, de modo que el discurso de éstos/as sea fluido, no se sientan extraños ni evaluados y que los sentimientos normalmente velados afloren a la mirada y comprensión adulta. Al respecto, algunos autores (Gunter \& Furnham, 1998; Threlfall, 1999) respaldan la técnica como apropiada en estudios sobre publicidad y convicciones o ideas compartidas, resaltan también que las niñas tienden a ser más colaborativas que los varones, aunque éstos últimos son más competitivos en situación de grupo y les agrada mostrar sus sentimientos, convicciones e ideas (Ruyter \& Scholl, 1998; Threlfall, 1999).

3. Para describir el SSA y la efectividad de las tácticas de manipulación (AM) empleadas por los niños para influir en sus padres a conseguir el producto simbolizado, se estableció una sesión individual con los padres (promedio 8 minutos) aplicándose una guía de entrevista formada por 14 ítems, de los cuales siete evalúan la aquiescencia hijo/a vs padres. Las entrevistas tanto a los niños como a sus padres fueron desarrolladas alternativamente por un psicólogo, una enfermera comunitaria o una asistente social. Las respuestas registradas se agruparon en categorías conceptuales (arquetipos). La validez de contenido de las dos instrumentos 2. y 3. fueron evaluadas mediante juicio de expertos, resultando significativa la concordancia de valoración entre los tres jueces y en siete criterios $(\mathrm{p}<0,01)$. Adicionalmente, la validez de respuestas de los sujetos se evaluó mediante el grado de aquiescencia en siete ítems: niños vs padres (estudio piloto: nov 2014).

\section{Materiales}

Cuatro láminas publicitarias plastificadas a color $(21$ x 29,7 cm) con imágenes del producto simbolizado (yogur) en las marcas: A="yoleit", B="puravida", C="laive”, $\mathrm{D}=$ ="gloria". El producto es referente para estudios de respuestas simbólicas y hedonistas (Kähkönen et al., 1997) que por su bajo contenido en grasa es considerado saludable y su información verbal es innecesaria. 


\section{Procedimiento}

En la evaluación del SSA se aplicó la técnica de entrevista en pequeños grupos a fin de que el discurso de los participantes sea fluido, no se sientan extraños ni evaluados y que los contenidos normalmente velados afloren a la mirada y comprensión adulta sobre la niñez. Luego de mostrar los materiales a los niños fueron reconociendo inmediatamente el producto simbolizado y asociado a la marca, dando lugar a las interrogaciones relacionadas al hedonismo y las AM. La técnica estadística (prueba chi-cuadrado) se empleó para determinar la correspondencia entre la frecuencia de preferencias de marca vs decisión de compra, para evidenciar la interdependencia entre las reacciones de los padres y la concordancia entre las expresiones de los hijos/as, y para contrastar entre el número de hermanos y áreas; el procesamiento de datos fue a través del programa informático SPSS (v.18). El proyecto de investigación fue aprobado por la sub comisión de ética del Consejo de Investigación de la Universidad Nacional Agraria de la Selva, Perú (ene 2013).

\section{Resultados}

El SSA basado en un producto simbolizado de cuatro marcas de yogur están organizadas en tres factores: (a) simbolismo hedonista asociado, (b) sensibilidad por la publicidad televisiva y, (c) conciencia del contenido material de la publicidad. El primero, fue determinado mediante frecuencia de preferencias de marca y su correspondencia con la decisión de compra por los padres, quiénes complacían significativamente a los niños hasta el 71,2\% de la frecuencia de solicitudes de los hijos/as ( $<<0,031)$ cuyo simbolismo expresado y consentido por los padres está predominantemente orientada hacia la marca "D-G" (Tabla 1).

Tabla 1. Concordancia entre las frecuencias de elección del producto simbolizado (niños) y la decisión de compra por los padres, 2015-16 (N=52)

\begin{tabular}{|c|c|c|c|c|c|c|c|c|}
\hline \multirow{2}{*}{$\begin{array}{l}\text { Elección } \\
\text { (marca) }\end{array}$} & & & & & \multicolumn{4}{|c|}{$\mathbf{p}$} \\
\hline & A-Y & B-P & C-L & D-G & & & \multirow{3}{*}{18,359} & \multirow{3}{*}{0,031} \\
\hline A-Y & 1 & 1 & 1 & 1 & 4 & 7,7 & & \\
\hline $\mathrm{B}-\mathrm{P}$ & 1 & 2 & 1 & 3 & 7 & 13,5 & & \\
\hline C-L & 1 & 1 & 1 & 1 & 4 & 7,7 & & \\
\hline D-G & 1 & 2 & 1 & 33 & 37 & 71,2 & & \\
\hline Recuento & 4 & 6 & 4 & 38 & 52 & 100,0 & & \\
\hline Porcentaje & 7,7 & 11,5 & 7,7 & 73,1 & 100,0 & & & \\
\hline
\end{tabular}

A-Y, B-P, C-L, D-G: Diferentes marcas de yogur.

$\mathrm{X}^{2}=$ Prueba chi-cuadrado $(\mathrm{gl}=9$, sig. $\mathrm{p}<0,05)$

En relación a las dificultades de conciencia sobre el contenido material de la publicidad $(72,0 \%)$ es significativamente mayor en el área urbana que el rural pero sin distinción entre el género. 
Actitudes manipulativas: Desde la perspectiva de los padres las AM aparecen en los niños después de la negativa a otorgar el producto simbolizado y se agrupa en: "ponerse triste y llorar", "revolcarse o hacer pataletas", y "amenazar"; siendo la primera, el que tuvo mayor frecuencia y éxito (69,7\%). Evidenciándose la interdependencia significativa con las siguientes reacciones de los padres: (a) "tener sentimiento de culpa" y "comprar el producto simbolizado" en $75,8 \%$ de los padres (p<0,019), (b) "explicar", y (c) "ignorar"; siendo las de menor frecuencia el de ignorar $(9,0 \%)$ (Tabla 2). Existe alta discordancia entre las expresiones de los niños y las respuestas de los padres, cuya variación porcentual (VP) y dependencia en toda las categorías de respuesta no es significativa $(p<0,749)$; por ejemplo, ante extraños como es natural, a los niños no les agrada expresarse que "lloran" pero en realidad ésta actitud fue la predominante para manipular a sus padres ( $\mathrm{VP}=-76,9 \%$ ), además de exagerarlas aquellas respuestas que no fueron efectivas para conseguir los productos simbolizados; por ejemplo, las "pataletas" y el "revolcarse" o abstenerse a "rogar" $(\mathrm{VP}=100,0 \%)$.

Tabla 2. Frecuencia de niños y padres involucrados en el simbolismo social y las actitudes manipulativas, 2015-16 $(\mathrm{N}=52)$

\begin{tabular}{|c|c|c|c|c|c|c|c|c|c|}
\hline Participantes & SSA & $\%$ & $\begin{array}{l}\text { Actitudes manipulativas } \\
\text { (niños) }\end{array}$ & $\mathrm{N}^{\circ}$ & $\%$ & $\begin{array}{c}\mathrm{N}^{\circ} \\
\text { hnos }\end{array}$ & $\begin{array}{l}\text { Reacción de los } \\
\text { padres }\end{array}$ & $\mathrm{N}^{\circ}$ & $\%$ \\
\hline $\begin{array}{l}\text { 1er. Grupo } \\
\begin{array}{l}(2015) \\
\mathrm{N}=25\end{array}\end{array}$ & 15 & 60,0 & Ponerse triste, llorar & 23 & 69,7 & $0-2$ & $\begin{array}{c}\text { Sentimiento de } \\
\text { culpa, compra el pro- } \\
\text { ducto simbolizado }\end{array}$ & 25 & 75,8 \\
\hline $\begin{array}{l}\text { 2do.Grupo } \\
\qquad \begin{array}{c}(2016) \\
\mathrm{N}=27\end{array}\end{array}$ & 18 & 66,7 & $\begin{array}{c}\text { Revolcarse, hacer pata- } \\
\text { letas }\end{array}$ & 7 & 21,2 & 3 & Explica & 5 & 15,2 \\
\hline Total: 52 & 33 & 63,5 & Amenzar & 3 & 9,1 & $4-5$ & Ignora & 3 & 9,0 \\
\hline
\end{tabular}

$\mathrm{SSA}=$ simbolismo social asociado; hnos $=$ hermanos de los niños evaluados

Contrastando las modalidades de reacción de los padres en función del número de hermanos de los niños: (a) tener sentimiento de culpa y comprar el producto simbolizado, (b) explicar y, (c) ignorar; encontramos tres situaciones predominantes: i) aplicar la reacción a) en $75,8 \%$ de las madres cuando los niños son hijos únicos o tienen entre 1 y 2 hermanos, ii) aplicar la reacción b) cuando tienen tres hermanos, y iii) aplicar la reacción c) cuando tienen entre 4-5 hermanos. Tanto la frecuencia de reacción de los niños (tristeza y llanto, pataletas y ruego) como las reacciones de las madres (ignorar, explicar, tener sentimiento de culpa y comprar) no muestran diferencias significativas entre áreas geográficas $(\mathrm{p}<0,908 ; \mathrm{p}<0,765)$.

\section{Discusión}

Determinada las características que representan al SSA y a su correlato las AM en grupo de niños de nueve años de edad con desarrollo intelectual adecuado diferenciados según el área, el género y número de hermanos. Se vislumbra la convergencia entre los factores simbolismo hedonista asociado y la conciencia del contenido material de la publi- 
cidad; evidenciándose en el primer caso la asociatividad a la marca por influencia directa en más de la mitad de sujetos (71,2\%) superando con 23,3\% de variación respecto a los hallazgos de Aktaş (2006) cuya frecuencia fue de 40,3\%; coincidiendo en cambio con la frecuencia de niños turcos que exigían a sus padres únicamente productos exhibidos en la televisión.

Estimándose por ello que en niños peruanos de nueve años de edad a pesar de poseer un desarrollo cognitivo adecuado, el SSA ocurre en similar magnitud a los de ocho o menores años de edad que aún no comprendían plenamente la intención persuasiva de la publicidad (Selman \& Byrne; 1974; Šramová, 2014).

Haciendo presumir que el consumismo de marcas continúa afirmándose conforme los argumentos de Neme y Rodríguez (2013) y manteniéndose vigente el simbolismo en niños como un modo de relacionamiento y el reconocimiento social como una necesidad (Holbrook, 1987; O'shaughnessy \& O’shaughnessy, 2012). Coincidiendo estos hechos con los reportes de varios autores para niños menores de nueve años, donde el simbolismo estaba reforzado por la influencia relacionada a la opinión de los pares y el apego significativo entre ellos (Neme \& Rodríguez, 2013; Raba’ah et al., 2014; Tsai, 2005). Así tenemos, más de las $3 / 4$ partes del grupo de niños peruanos elegían la misma marca que preferían los pares $(78,5 \%)$ y que los padres complacían hasta el $71,2 \%$ de las solicitudes predefinidas por los hijos, resultados que tienen correspondencia con las afirmaciones de Raba'ah et al. (2014) para niños menores. Surgiendo en dicha interrelación una influencia emocional significativa basada en la AM consciente hasta obtener el producto simbolizado, que está confirmando la interdependencia con las respuestas de los padres, de tener sentimiento de culpa por el rechazo dado, optar por reconsiderar y llegar a proveer lo solicitado, en desventaja de "explicar" (15,2\%) e "ignorar" (9\%); y que las tácticas de los niños, por ejemplo, de "ponerse triste y llorar" tenían mayor éxito (69,7\%).

Entre tanto, la AM consciente de los niños determinada indirectamente, primero, en razón que es natural a los niños no les agradaba declarar que lloran, cuando en realidad desde la perspectiva de los padres ésa actitud fue la táctica manipulativa predominante y eficaz ( $\mathrm{VP}=-76 \%)$; segundo, por la exageración de las tácticas menos efectivas para lograr el producto simbolizado ( $\mathrm{VP}=100,0 \%)$; y tercero, por la abstención a declarar otras tácticas. Afianzándose en la proposición de que las AM en los niños para lograr un producto es consciente y surgen durante el seguimiento de conductas a los padres (Berne, 2011; Dotsenko, 2003; Monich \& Matveeva, 2012; Zhumagaliyeva \& Barabanova, 2014).

En relación a la variabilidad en la tolerancia de los padres sobre el comportamiento hedonista y AM de los niños según el número de hermanos, que ocurre mayormente cuando son hijos únicos o con 1-2 hermanos (75,8\%), la comparación aunque no exista diferencias según área geográfica y a la falta de referencias base para el número de hermanos, concuerda con Soenens y Vansteenkiste (2010) quiénes sostienen: dada la interacción interpersonal la AM o coacción psicológica consiente surge cuando los niños tienen necesidad de conseguir un objetivo deseado, y en cada cultura las características de tolerancia y la AM pueden variar según el tipo de familia, la edad y el género (Menelaos, 2015; Soenens \& Vansteenkiste, 2010).

Sobre las dificultades para el reconocimiento del contenido material (tercer factor del SSA) que ocurría en la mayoría de niños $(72,5 \%)$, existe correspondencia con los argumentos de Elliot (2014), en que los contenidos simbólicos de la televisión generan comportamientos de compra más hedonista, debido al alto nivel de materialismo que influyen 
negativamente en la orientación de los valores y la AM de los niños (Šramová, 2014, 2014a). Revelándose que aún a la edad de nueve años los niños peruanos no comprenderían plenamente la intención persuasiva de la publicidad y el uso de AM es mayormente para conseguir el objetivo deseado. Evidencias que se oponen a los reportes de Selman y Byrne (1974), quiénes señalan que el SSA y la AM ocurría en niños de ocho años y menores. Así tenemos que la mayoría de niños peruanos (84\%) afirmaban entre otros aspectos, que la publicidad televisiva "no mentía" o que "decía cosas buenas" (72\%); y con mayor intensidad en los niños del área urbana $(\mathrm{p}<0,011)$ reflejando la característica común de una cultura de consumo dada a través del SSA (Fenollar y Ruiz, 2006; Tsai, 2005).

Sobre las diferencias del SSA y AM según área geográfica, varía con los reportes en Santiago de Chile (Uribe et al., 2006) y en Londres (Goldstein, 1994), donde los padres de menor nivel socioeconómico poseían una visión poco más positiva de la publicidad; frente al $52 \%$ de padres de familia del área rural en estudio, que eran más restrictivos para la visualización televisiva de sus hijos; lo cual es consistente con los hallazgos en padres del grupo social medio-bajo, que se mostraban desconfiados hacia la publicidad porque percibían como una influencia negativa (Aktaş, 2006; Goldstein, 1994; González, 2008; Šramová, 2014a). Sosteniéndose que en cada grupo social la magnitud del impacto negativo de la publicidad es diferente y el nivel socioeconómico de los padres sería un factor mediador importante para caracterizar el SSA.

Limitaciones: dado que el estudio surge a partir de un programa de atención primaria de salud, y el objetivo es verificar el constructo teórico sobre el desarrollo de la capacidad de comprensión del contenido material de la publicidad, en una edad específica y en base a un producto simbolizado, los resultados no representan a la población de niños del país. Sin embargo, no por ello son generalizables aunque sería oportuno confirmar los hallazgos en muestras mayores. A pesar de sus limitaciones el estudio tiene ventajas metodológicas: (a) grupo de estudio homogéneo determinado mediante desarrollo cognitivo intelectual, (b) participación de los padres y, (c) comparación socioeconómica (área geográfica y número de hermanos).

Aportes: Se revela que aún a la edad de nueve años los niños no comprenden plenamente la intención persuasiva de la publicidad y usan tácticas manipulativas para conseguir el objeto simbolizado. Para el país, comparando los aspectos psicosociales del desarrollo y la salud mental infantil según variables socioeconómicas, el estudio es único en su naturaleza, una actividad prioritaria de la educación para la salud que contribuirá en la implementación multidisciplinaria de programas de desarrollo emocional en la actitud crítica ante los medios de comunicación, en la protección de consecuencias de las actitudes simbólicas y la AM, en la autoestima y en los valores. Frente a deslindes teóricos limitados en diversas cuestiones del simbolismo social y la presencia de la AM, el estudio propone orientaciones de la educación para la salud, en un contexto donde los padres viven en áreas geográficas diferentes. Se recomiendan: a) desarrollar programas de alfabetización mediática orientadas a la familia y a la escuela, donde los profesores ayuden comprender a los niños la intención persuasiva de la publicidad y el equilibrio entre la necesidad y la salud; b) se regulen y se promocionen las mejores prácticas en la publicidad infantil; c) las iniciativas en salud pública presten mayor atención los aspectos simbólicos de los alimentos versus cualidades nutricionales y; d) incluir en futuras investigaciones la religión de los padres como una variable moderadora. 


\section{Conclusiones}

El SSA está presente en la mayoría de niños de nueve años de edad de la Amazonía peruana con nivel cognitivo intelectual adecuado para la edad. Las AM conscientes para conseguir el producto simbolizado continúan intensificándose como un simbolismo transformado en un modo de relacionarse y con el reconocimiento social afirmándose como necesidad reforzada por los pares. Existen dificultades para comprender plenamente el contenido material de la publicidad. La frecuencia del SSA y la AM no difieren según el género ni entre el área geográfica, excepto en las dificultades de comprensión del contenido material de la publicidad, que ocurre con mayor intensidad en el área urbana. En cambio hay variabilidad según el número de hermanos, tanto en actitudes de tolerancia de los padres al comportamiento hedonista como en las tácticas manipulativas de los niños.

\section{Referencias}

Abregú, L.F. (2009). Evolución del razonamiento analógico en niños: seguimiento desde los seis hasta los once años de edad. Avances en Psicología Latinoamericana, 27(1), 97-110.

Addis, M. \& Holbrook, M. B. (2006). On the conceptual link between mass customisation and experiental consumption: an explosion of subjectivity. J. Consumer Behav, 1(1), 50-66.

Aktaş, Y. (2006). The effects of television food advertisement on children's food purchasing requests. Pediatr. Int, 48(2), 138-145.

American Psychiatric Association (APA). (2013). Intellectual disability fact sheet-DSM-5. Arlington, USA: Author.

Anzieu, D. (1981). Para una psicolingüística psicoanalítica: breve balance y cuestiones preliminares. En D. Anzieu, B. Gibello, R. Gori et al. (Eds.), Psicoanálisis y lenguaje, del cuerpo a la palabra (pp. 1-23). Buenos Aires: Kapelusz.

Barbeta, M. (2015). El símbolo da qué pensar: esbozo para una teoría psicosociológica del simbolismo. Perspectiva cognitivo-afectiva, discurso e interpretación. Sociológica, 30(85), 163-196.

Bart, S. \& Maarten, V. (2010). A theoretical upgrade of the concept of parental psychological control: Proposing new insights on the basis of self-determination theory. Developmental Review, 30(1), 74-99.

Bereczkei, T. (2015). The manipulative skill: Cognitive devices and their neural correlates underlying Machiavellian's decision making. Brain and Cognition, 99(1), 24-31.

Berne, E. (2011). Games people play: The psychology of human relationships. Moscow: Eksmo.

Carreño, L. (2010). El desarrollo de las competencias emocionales como medio para promover la conciencia crítica ante los medios de comunicación. [Comunicación Científica]. En la Universidad Autónoma de Barcelona (Ed.), Memorias, I Congreso Euro-Iberoamericano "Alfabetización mediática y culturas digitales", may 13-14 (pp.1-15).: Sevilla, España: GCE.

CIE-11. (2015). The international classification of diseases 11th revision. Manchester, UK: WHO-FIC Network Meeting.

Carona, A., Weiss, B., Harris, V., \& Catron, T. (2006). Parenting behavior dimensions and child psychopathology: specificity task dependency and iterative relations. Journal of Clinical Child and Adolescent Psychology, 35(1), 34-45. 
Decety, J., Jackson, P., Sommerville, J., Chaminade, T., \& Meltzoff, A. (2004). The neural bases of cooperation and competition: An fMRI investigation. NeuroImage, 23(1), 744-751.

Derbaix, C. \& Bree, J. (1997). The impact of children's affective reactions elicited by commercials on attitudes toward the advertisement and the brand. Intern. J. of Research in Marketing, 14(3), 207-229.

Dotsenko, E. L. (2003). Psychology of manipulation. Moscow: Rech.

Douglas, M. \& Baron, I. (1990). El mundo de los bienes: hacia una antropología del consumo. México: Grijalbo.

Elliot, C. (2014). Food as people: Teenagers' perspectives on food personalities and implications for healthy eating. Soc. Sci. \& Med, $121(1), 85-90$.

Fenollar, P. \& Ruiz, S. (2006). La posesión de productos con significado social para el consumidor. Determinantes internos y externos. Revista Española de Investigación de Marketing. ESICREIMKE, 10(2), 7-24.

Furth, H. (1992). El conocimiento como deseo. Un ensayo sobre Freud y Piaget. Madrid: Alianza.

Gállego, J., Aliaga, P., \& Granizo, C. (2014). Promoción de la salud y educación para la salud en los centros educativos. En AM Palmar (Ed.), Métodos educativos en salud (pp. 153-174). Barcelona: Elsevier Spain.

Groldstein, J. (1994). Children and advertising: policy implications of scholarly research. Londres: The Advertising Association.

González, C. (2008). La categoría de producto y el mensaje transmitido en la publicidad infantil de alimentos. Revista Latina de Comunicación Social, 63(1), 480-491.

Gunter, B. \& Furnham, A. (1998). Children as consumers: A psychological analysis of the young people’s market. London: Routledge.

Hastings, G., Stead, M., McDermott, L., Forsyth, A., MacKintosh, A., Rayner, M. et al. (2003). Review of research on the effects of food promotion to children. Final report. Glasgow (Scotland, UK): University of Glasgow - CSM, FSA.

Holbrook, M. B. (1987). Mirror, mirror, on the wall, what on advertising. J. Mark, 5 1(7), 95-103.

Huber, L. (2002). Consumo, cultura e identidad en el mundo globalizado. Estudios de caso en los Andes. Lima: IEP.

Islas, P., Pérez, A. y Hernández, G. (2015). Rol de enfermería en educación para la salud de los menonitas desde el interaccionismo simbólico. Enfermería Universitaria, 12(1), 28-35.

Kähkönen, P., Tuorila, H., \& Lawless, H. (1997). Lack of effect of taste and nutrition claims on sensory and hedonic responses to a fat-free yogurt. Food Qulity and Prejtience, 8(2), 125-130.

Kunkel, D. (2010). Mismeasurement of children's understanding of the persuasive intent of advertising. J Child Media, 4(1), 109-117.

Lawlor, M. A. \& Prothero, A. (2010). The established and potencial mediating variables in the child's understanding of advertising intent: Towards a research agenda. J Market Manag, 18(1), 48199.

Lévi-Strauss, C. (1987). Antropología estructural. Barcelona: Paidós.

Malinowski, B. (2001). Los argonautas del Pacífico occidental. Barcelona: Península.

Marcos, E. \& Carrillo, C. (2014). Aspectos psicosociales del cuidado al niño y al adolescente. En G. Granados (Ed.), Aplicación de las ciencias psicosociales al ámbito del cuidar (pp.151-171). 
Barcelona: Elsevier Spain.

Martínez, J. R. (2014). Investigación enfermera en atención primaria: prioridades. En J. R. Martínez y R. Del Pino (Eds.), Manual práctico de enfermería comunitaria (pp. 385-390). Barcelona: Elsevier Spain.

Menelaos, A. (2015). I am right for your child! Tactics for manipulating potential parents-in-law. Hum Nat, 26(1), 378-391.

Monich, M. S. \& Matveeva, L. V. (2012). Personality determinants of manipulative behavior in the negotiation process. Psychology in Russia: State of the Art, 5(1), 314-332.

Nefat, A. \& Dujmović, M. (2012). Children's advertising on television and their consumer socialization: parents' attitudes. Ekon. Istraz., 25(1), 145-146.

Neme, S. R. \& Rodríguez, L. (2013). Consumo simbólico: una perspectiva sociocultural en la comprensión del comportamiento del consumidor. Revista Iberoamericana de Psicología: Ciencia y Tecnología, 6(2), 27-33.

Nunes, M. (1998). Evolução da relação de comunicação entre a criança e a televisão. Ver Bras de Ciên da Com, $21(2), 93-113$.

Ortí, A. (1994). La estrategia de la oferta en la sociedad neocapitalista de consumo: Génesis y praxis de la investigación motivacional de la demanda. Política y Sociedad 16(1), 37-92.

O’Shaughnessy, J. \& O’Shaughnessy, N. J. (2002). Marketing, the consumer society and hedonism. Eur. J. Market, 36(5/6), 524-547.

Programa de Naciones Unidas para el Desarrollo - PNUD. (2013). Informe sobre desarrollo humano. Perú 2013. Lima: UNDAF Peru.

Petty, R. E. (2006). A metacognitive model of attitudes. J Consum Res, 33(1), 22-24.

Piaget J. (1970). The stages of the intellectual development of the child. En Mussen PH, Conger J. J, Kagan J. Readings in child development and personality (pp. 291-298). New York: Harper \& Row.

Raba'ah, S., Turinam, S., Hamzah, A., \& Tamam, E. (2014). The influence of religiosity, parental and peer attachment on hedonistic behavior among Malaysian youth. Procedia Soc Behav Sci, 122(1), 393-397.

Ramasco, M. (2014). Promoción de la salud y educación para la salud. En A. M. Palmar (Ed.), Métodos educativos en salud (pp. 1-19). Barcelona: Elsevier Espain.

Ramos, A. J., Fernández, S., \& Carranza, E. M. (2014). Educación para la salud. En J. R. Martínez y R. Del Pino (Eds.), Manual práctico de enfermería comunitaria (pp. 197-203). Barcelona: Elsevier Spain.

Raven, J. C. (2000). The Raven's progressive matrices: Change and stability over culture and time. Cognitive Psychology, 41(1), 1-48.

Ruiz, V. (2014). Salud escolar: la enfermera escolar. En J. R Martínez y R Del Pino (Eds.), Manual práctico de enfermería comunitaria (pp. 585-589). Barcelona: Elsevier Spain.

Rozendaal, E., Buijzen, M., \& Valkenburg, P. (2011). Children's understanding of advertisers' persuasive tactics. Int J Advert, 30(2), 329-250.

Ruyter, K. \& Scholl, N. (1998). Positioning qualitative market research: reflections from theory and practice. Qual Market Res Int J, 1(1), 7-14.

Schutz, A. (1974). El problema de la realidad social. Buenos Aires: Amorrortu. 
Soenens, B. \& Vansteenkiste, M. (2010). Theoretical upgrade of the concept of parental psychological control: Proposing new insights on the basis of self-determination theory. Developmental Review, 30(1), 74-99.

Salvador, L., Reed, G., Vaez, L., Cooper, S., Martínez, R., Bertelli, M. et al. (2011). Intellectual developmental disorders: towards a new name, definition and framework for "mental retardation/ intellectual disability” in ICD-11. World Psychiatry, 10(3), 175-280.

Selman, R. L. \& Byrne, D. F. (1974). A structural-developmental analysis of levels of role taking in middle childhood. Child Dev, 45(1), 803-806.

Soto, E. (2010). Nivel de conocimiento de los padres de familia acerca de la salud mental de sus hijos preescolares en una institución educativa de Lima. Tesis de licenciatura, Facultad de Enfermería, Universidad Nacional Mayor de San Marcos. Lima. Recuperado de http://cybertesis. unmsm.edu.pe/handle/cybertesis/520

Šramová, B. (2014). Aggressive marketing, consumer kids and stereotyping of media contents. Procedia Soc Behav Sci, 140(1), 255-259.

Šramová, B. (2014a). Media literacy and marketing consumerism focused on children. Procedia Soc Behav Sci, 141(1), 1025-1030.

Threlfall, K. D. (1999). Using focus groups as a consumer research tool. J Market Pract Appl Market Sci, 5(4), 102-105.

Tsai, S. (2005). Utility, cultural symbolism and emotion: A comprehensive model of brand purchase value. Intern. J. of Research in Marketing, 22(1), 277-291.

Uribe, B. R., Acuña, M. C., Carrasco, A., \& Checura, S. (2006). Actitud parental hacia la publicidad infantil en Chile: el rol del GSE y de la dicotomía urbano-rural. Estudios de Administración, $13(2), 25-46$.

Wartella, E. (2009). Cognitive and affective factors of tv advertising's influence on children. West J Speech Comm, 48(1), 171-183.

Wilson, C., Grizzle, A., Tuazon, R, Akyempong, K., \& Cheung, C. (2011). Media and information literacy. Curriculum for teachers. Paris: UNESCO.

World Health Organization (WHO). (2010). Set of recommendations on the marketing of foods and non-alcoholic beverages to children. Switzerland: Author.

Young, B. (2015). Does food advertising influence children's food choices? A critical review of some of the recent literature. Int J Advert, 22(1), 441-459.

Zhumagaliyeva, B. \& Barabanova, E. (2014). Features of manipulative behavior in operational officers' professional activity. Procedia-Social and Behavioral Sciences, 140(1), 9-14. 


\section{LUIS FIDEL ABREGÚ TUEROS \\ http://orcid.org/0000-0002-0938-5061}

Psicólogo, profesor investigador en Área de Desarrollo Humano, Universidad Nacional Agraria de la Selva, Perú (Reg. Concytec N 17642). Mestrado em Psicología Social e Institucional (Universidade Federal do Rio Grande do Sul, Brasil) y doctor en Salud Pública (Universidad Nacional "Federico Villareal", Lima, Perú). Profesor visitante en Universidad de Alcalá, España.

Dirección institucional: Universidad Nacional Agraria de la Selva, Área de Desarrollo Humano. Av. Universitaria Km 1,5. Cód. Postal 10131. Tingo María, Perú.

E-mail: luis.abregu@,unas.edu.pe

\section{JUSSARA MARÍA ROSA MENDES \\ https://orcid.org/0000-0002-3908-4964}

Doutora em Serviço Social (Pontifícia Universidade Católica de São Paulo). Pós doutorado em Serviço Social (Universität Kassel, Alemanha. Docente credenciada ao Programa Pós-graduação em Psicologia Social e Institucional do Instituto de Psicologia (Universidade Federal do Rio Grande do Sul).

E-mail: jussaramaria.mendes@gmail.com

\section{LOURDES ABREGÚ ARROYO \\ http://orcid.org/0000-0001-8101-2764}

Enfermera asistencial, Programa de Crecimiento y Desarrollo en el Escolar y Adolescente, Hospital Nacional EsSalud "E. Rebagliati” Lima (Perú). Especialista en Enfermería (Universidad Peruana Cayetano Heredia, Perú).

E-mail: mikita0311@,hotmail.com

\section{Histórico}

Contribuição dos autores

Financiamento
Submissão em: 27/05/2016

Aceite em: 08/02/2019

Concepção: LFAT; LAA

Coleta de dados: LFAT; JMRM, LAA

Análise de dados: LFAT

Elaboração do manuscrito: LFAT; JMRM; LAA

Crítico revisões de conteúdo intelectual importante: LFAT; JMRM

Aprovação final do manuscrito: LFAT; JMRM;

LAA

El trabajo fue apoyado parcialmente por la OEA-OPS y se recibió una bolsa de estudios de CAPES a través del Convenio OEA-GCUB.

Consentimento de uso de imagem Não se aplica 